

\title{
El conocimiento del entorno en Educación Infantil. Teoría y práctica desde las Ciencias Sociales y su didáctica
}

\author{
Knowledge of the environment in Early \\ Childhood Education. Theory and practice \\ from the Social Sciences and their teaching
}

DOI: $10.7203 / D C E S .40 .16962$

\author{
Mercedes Millán Escriche \\ Universidad de Murcia, memillan@um.es \\ ORCID iD: http://orcid.org/0000-0001-6833-7556
}

\begin{abstract}
RESUMEN: La posibilidad de incluir, en Educación Infantil, conocimientos relacionados con Ciencias Sociales, como el espacio y el tiempo, ha venido encontrando opiniones diversas, incluso para el segundo ciclo. Sin embargo, actualmente se acepta que no solo es pertinente, sino inexcusable para iniciar una progresión satisfactoria que mejore resultados en las etapas posteriores y que el verdadero reto para los docentes es aplicar los métodos y las estrategias apropiados. Así se viene considerando en la formación de futuros docentes con la asignatura Ciencias Sociales y su Didáctica en Educación Infantil impartida en la Universidad de Murcia. De ello trata este trabajo, cuyo objetivo principal es poner de manifiesto la diversidad de actividades y recursos didácticos que el alumnado de dicha asignatura ha sido capaz de elaborar con el fin de ser utilizados en los centros educativos, destacando, en consonancia con la normativa vigente, los relativos al área de Conocimiento del Entorno.
\end{abstract}

Palabras Clave: currículo, Educación Infantil, conocimiento del entorno, didáctica de las Ciencias Sociales, teoría y práctica

ABstract: The possibility of including, in Early Childhood Education, knowledge related to Social Sciences, such as space and time, has been finding diverse opinions, even for the second cycle. However, it is currently accepted that it is not only pertinent, but inexcusable to initiate a satisfactory progression that improves results in the later levels and that the real challenge for teachers is to apply the appropriate methods and strategies. This is being considered in the training of future teachers with the subject Social Sciences and their teaching in Early Childhood Education taught at the University of Murcia. This is what this work is about, the main objective of which is to highlight the diversity of didactic activities and resources that the students of this subject have been able to develop in order to be used in educational centers, highlighting, in line with current regulation, those relating to the area of Environmental Knowledge.

KEYWORDS: curriculum, Early Childhood Education, Environmental Knowledge, teaching of the Social Sciences, theory and practice

Fecha de recepción: abril de 2020

Fecha de aceptación: octubre de 2020 


\section{INTRODUCCIÓN/JUSTIFICACIÓN}

Esta aportación surge desde la convicción personal de la importancia de conocer, valorar y respetar nuestro entorno desde las edades más tempranas. Sin duda, ha contribuido a ello la propia formación y la experiencia docente e investigadora, que ha derivado en un aprendizaje sobre el entorno, entendido este desde una perspectiva amplia, puesto que, como apuntan Souto y Monteagudo (2016), los aprendizajes son responsabilidad del individuo, pero no puede obviarse que se realizan en el seno de una comunidad social y cultural y, por lo tanto, pueden considerarse el resultado de una interrelación entre el sujeto y el contexto social, ese que constituye el primer entorno del alumnado y que no es solamente la parte visible sino la que forja una representación mental del territorio en un individuo a partir de lo que percibe. Una percepción que es particular, subjetiva, emocional e influenciada por los prejuicios culturales que inundan la experiencia vital de las personas.

Todo ello se relaciona con las pretensiones puestas en la asignatura Ciencias Sociales y su Didáctica en Educación Infantil, en la que el alumnado, que cursa estos estudios en la Universidad de Murcia, debe alcanzar una serie de competencias. Entre estas cabe destacar las siguientes:

1. Conocer los fundamentos científicos y tecnológicos necesarios para desarrollar los objetivos del currículo de Educación Infantil relacionados con las Ciencias Sociales.

2. Conocer las teorías que explican la adquisición y desarrollo del aprendizaje de contenidos científicos y sociales.

3. Adquirir conocimientos sobre la evolución del pensamiento, las costumbres, las creencias y los movimientos sociales y políticos a lo largo de la historia.

4. Conocer los momentos más sobresalientes de la historia de las ciencias y la técnica y su trascendencia a lo largo de la historia humana.

5. Elaborar propuestas didácticas en relación con las interacciones ciencia, tecnología, sociedad y desarrollo sostenible.

6. Promover el conocimiento, interés y respeto por el medio natural, social y cultural a través de proyectos didácticos adecuados.

7. Fomentar experiencias de iniciación a las tecnologías de la información y la comunicación.

FIGURA 1. Normativa vigente para Educación Infantil en la Región de Murcia



Fuente: Elaboración propia 
Estas competencias encuentran estrecha relación con el marco normativo vigente (figura 1) para esta etapa educativa. Se trata del Decreto 254/2008, de 1 de agosto, por el que se establece el currículo del Segundo Ciclo de la Educación Infantil en la Comunidad Autónoma de la Región de Murcia. Su Artículo 5. Áreas, remite al artículo 6 del Real Decreto 1630/2006, de 29 de diciembre, que determina la organización del currículo del segundo ciclo de la Educación Infantil en las siguientes áreas: Conocimiento de sí mismo y autonomía personal. Conocimiento del entorno. Lenguajes: Comunicación y representación.

Es importante destacar cómo la normativa pone de relieve que observar y explorar su entorno familiar, natural y social, así como descubrir y valorar el entorno natural más próximo, e interesarse por algunas de las principales manifestaciones culturales y artísticas de la Región de Murcia, constituyen objetivos a alcanzar en esta etapa. Asimismo, que las orientaciones metodológicas, presentes en dicha normativa, han constituido un elemento primordial para desarrollar la parte práctica de la asignatura, pero sin obviar que, en los futuros docentes, no debe faltar la formación teórica en las ciencias que se tratan en ella.

Con el Área Conocimiento del Entorno, las más próxima a las enseñanzas de las Ciencias Sociales, siempre considerando que sus contenidos adquieren sentido desde la complementariedad con el resto, se pretende favorecer en niños y niñas, de manera reflexiva y participativa, el proceso de descubrimiento y representación de los diferentes contextos que componen el entorno infantil, así como facilitar su inserción en ellos.

Referirse al entorno, desde esa perspectiva amplia, ya aludida, responde a un conjunto de circunstancias o factores sociales, culturales, morales, económicos, ambientales, que rodean a una persona o a una sociedad y que incide en su estado o desarrollo. Por ello es importante la relación con el tiempo y, en este sentido, las competencias 3 y 4 obligan a conocer nuestro pasado, el qué y el cómo ha sido el devenir de quienes nos han precedido, cuál ha sido el patrimonio que nos han legado sobre sus costumbres, creencias, modelos de organización, tipologías de asentamiento,..., así como estar al tanto de los momentos más relevantes de la ciencia y la tecnología y su repercusión para el ser humano, aspectos estos últimos que deben conseguirse como competencia 5.

El espacio se refiere aquí al entorno en su conjunto, ya sea natural o sociocultural, y debe constituir un elemento clave en la formación del alumnado de esta asignatura, tal y como se pretende alcanzando las competencias 6 y 7, puesto que, mediante las estrategias didácticas adecuadas, les capacitará para que, en su futuro como docentes, contribuyan al desarrollo de los niños y propicien su aproximación a la interpretación del mundo.

La normativa citada anteriormente, en la que es obligatorio fijarse, ya apunta que, en su práctica docente, los maestros deberán utilizar métodos de trabajo sustentados en experiencias, actividades y juegos, puesto que va ser así como se impulse un ambiente de afecto y confianza entre todos los participantes. Por lo tanto, para la formación que se pretende, puede considerarse la idoneidad de los contenidos de la asignatura Ciencias Sociales y su Didáctica en Educación Infantil, así como las prácticas de clase y el trabajo de curso que se concretan en propuestas de actividades empíricas de ejercicios y resolución de problemas, utilizando para ello distintos métodos como estudio de casos, aprendizaje orientado a proyectos, etc., así como exposiciones, debates y simulaciones.

\section{EL ESTADO DE LA CUESTIÓN}

Como se desprende de lo antedicho, la complementariedad y el carácter integrador impregnan la filosofía del currículo de la etapa de Educación Infantil y, por ello, resulta irrenunciable la utilización en la práctica de distintos contenidos correspondientes a las tres áreas que lo articulan.

Así se ha ido avanzando paulatinamente para su aplicación desde que, a principios de los ochenta, se iniciase la Reforma de Educación Infantil, sobre todo desde la Orden Ministerial del 26 de abril de 1985 (Alonso, 2007) y el Programa Experimental para la Reforma de Educación Infantil. 
Poco después, como apunta Gervilla (2016), el Diseño Curricular Base, establecido por el Ministerio de Educación en 1989, adelantaba los criterios que siguen estando vigentes actualmente, considerando que, en Educación Infantil, es necesario aprovechar las posibilidades de desarrollo del alumnado potenciándolas y afianzándolas mediante la acción educativa y, asimismo, impulsando el alcance de las competencias, destrezas, hábitos y actitudes que les permitirán una mejor adaptación a la Educación Primaria, puesto que los logros y experiencias de la infancia son constituyentes del posterior "si mismo" (Egan, 1991). Todo ello sin olvidar que el código pedagógico del entorno es un producto mixto, complejo y contradictorio, de discursos y prácticas, que se muestra muy resistente a los caprichos normativos (Mateos, 2008).

Los docentes deben conocer la sociedad en la que se encuentran inmersos, pero sin que esto signifique ese conocimiento consista en empaparse de los lugares de la "memoria oficial" del grupo, ni en los lugares de su identidad territorial. Se trata fundamentalmente de conseguir enriquecerse de forma progresiva y, como señalan Romero y Luis (2008),

Llegar a comprender las circunstancias que modelan nuestra existencia en colectividad, la gradual apropiación reflexiva de las condiciones de la acción en nuestro mundo, en tanto que requisito imprescindible -no suficiente- para la implicación e intervención en las dimensiones compartidas de la actividad social. (p. 15)

Espacio y tiempo son campos de actuación docente indispensables desde edades tempranas. En este contexto, según Schiller y Rossano (1993), el profesorado puede aprovechar las diferentes oportunidades, que se presentan a lo largo del día, para desarrollar habilidades y conceptos que ampliarán, en el alumnado de Educación Infantil, su comprensión del propio entorno, puesto que las experiencias que vive cotidianamente, en dicho espacio, viabilizan su toma de conciencia de la distancia que lo separa de objetos y personas, lo cual es básico para la construcción de nociones espaciales vinculadas al conocimiento del entorno (García, Villegas y González, 2015).

Respecto a las cuestiones temporales, Rivero y Pelegrín (2019) señalan que no existe una regulación explícita de la Historia en Educación Infantil por parte del currículo, pero que resulta evidente la relevancia y significación del pensamiento histórico cuando se trata de formar a los docentes de esta etapa, ya que sí que configuran los contenidos que podrán impartir a sus futuros alumnos. En la práctica, sabemos que deberán recurrir a estrategias bien diversas y Miralles y Rivero (2012) indican que el papel del docente no debe ser el de un mero transmisor de conocimientos, sino el de facilitador de los aprendizajes.

Para familiarizarse con las cuestiones temporales, entre otras propuestas innovadoras, destacan la importancia de la narración, puesto que favorece ampliar las vivencias y despertar la imaginación. También contribuye a ello llevar a cabo una incursión en las propias experiencias cotidianas, como son sus ritmos vitales semanales, y mediante un acercamiento a su historia familiar. Los autores consideran, así mismo, la trascendencia del papel de la imagen en sus distintos formatos, ya que estos elementos visuales "permiten acceder al conocimiento de la historia por medio del sentido que más información hace llegar a los niños de esta edad” (Miralles y Rivero, 2012 p.84).

En esta etapa, los cuentos constituyen una estrategia eficaz y sus intereses pueden ser vinculados a la consecución de objetivos relacionados con diferentes ámbitos del entorno y con el desarrollo de actitudes personales (Marín-Díaz y Sánchez-Cuenca, 2015), tanto estos como el juego son aceptados por buena parte de los investigadores. En concreto, como dice López (2010) el juego,

se identifica con diversión, satisfacción y ocio, con la actividad contraria a la actividad laboral, que normalmente es evaluada positivamente por quien la realiza. Pero su trascendencia es mucho mayor, ya que a través del juego se transmiten valores, normas de conducta, resuelven conflictos, educan a sus miembros jóvenes y desarrollan muchas facetas de su personalidad (p.19). 
Puede añadirse que, desde los primeros años de la persona, el juego es un motor de progreso para la capacidad intelectual y, además, potencia otros valores humanos como son la afectividad, la sociabilidad y la motricidad, entre otros. En esencia, todo ello no queda muy alejado de lo que se pretende conseguir para desarrollar la personalidad de los niños, porque el juego se integra en su medio natural, social y cultural a partir de las relaciones de interdependencia que se establecen entre el sistema formal, cuyo ámbito es la escuela; el sistema no formal, integrado por la familia, las asociaciones vecinales, el barrio... y el sistema informal que está constituido por la ciudad y el paisaje (García-Ruiz, 2013). En este contexto, Pluckrose (2002) considera lo siguiente:

Todos nosotros necesitamos desarrollar un sentido personal de identidad; comprender la sutil relación entre la familia, la comunidad y la nación; captar el modo en que las instituciones locales y nacionales han crecido y se han desarrollado; y apreciar las creencias que sustentan los valores y costumbres de la sociedad en cuyo seno hemos nacido (p. 21).

En definitiva, el entorno, que constituye el espacio vital del alumnado, puede relacionarse con el proceso cognitivo que le permitirá identificarse con otros miembros de su comunidad, puesto que con ellos comparte un simbolismo, una identidad, pero sin llegar a constreñir la amplitud de miradas hacia los otros, tal y como alerta López (2003). Se trata, en suma, de una experiencia que podemos asociar con la cultura, sin la que, según Bruner (1997), no se podría evolucionar mentalmente, ya que es la cultura la que aporta los instrumentos necesarios para organizar y entender el espacio social, utilizando para ello las herramientas de la misma cultura.

Atendiendo al currículo de la etapa, se convierte en un objetivo potenciar en el alumnado la observación y exploración de su entorno de forma activa, de manera que se genere la interpretación de algunas situaciones y hechos significativos. Por ello, los contenidos referentes a la formación del pensamiento social, al conocimiento de los fenómenos, sucesos, productos sociales y culturales, así como a la identificación de algunos cambios en el modo de vida, en relación con el paso del tiempo, provienen de la percepción global de lo social, lo espacial y lo temporal (Aranda, 2003) y constituyen los ejes vertebradores de las Ciencias Sociales.

FIGURA 2. Bloques del Área de Conocimiento del Entorno.

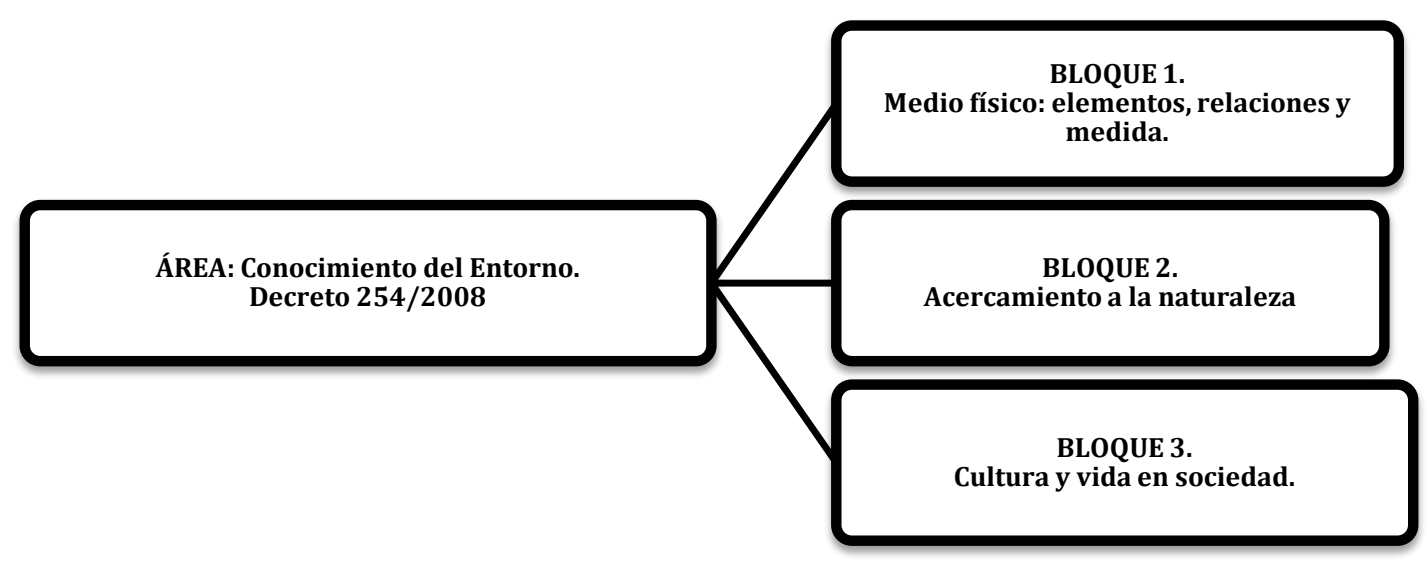

Fuente: Elaboración propia

De ahí que, para lograr resultados coherentes y evaluables, resulte necesario utilizar el método interrogativo que lleve a una reflexión a los futuros profesionales, acerca de los diferentes elementos que estructuran el currículo. Así, se preguntan Blanco, De la Calle, Fernández, et. al., (2008 p. 118) “¿Para qué sirven los aprendizajes sociales en este nivel de enseñanza? ¿Qué deben aprender los niños y niñas de esta etapa sobre las Ciencias Sociales? ¿Cómo aparecen estos contenidos en el currículo?”. Siendo capaces de responder a estas preguntas, los futuros maestros 
podrán tomar decisiones para enfocar el desarrollo curricular de esta etapa bajo una mirada crítica, apoyada en fundamentos científicos, psicológicos, sociales y metodológicos. En este sentido, por lo que respecta a las prácticas, para avanzar en el conocimiento del entorno, han sido muchos los autores que estiman necesario acercar al alumnado al contexto que es objeto de estudio, por ejemplo, descubriendo el patrimonio que les resulta más próximo de manera vivencial y, como dice Pagés (2002), estas prácticas,
Vienen, en parte, determinadas por el currículo y se caracterizan por las relaciones que en ellas se establecen entre el saber social (objeto de enseñanza y de aprendizaje), el alumnado (con sus representaciones previas del saber), y el profesorado (con sus ideas sobre el saber, la enseñanza y el aprendizaje). Buena parte de lo que ocurre en la práctica es consecuencia de las decisiones que el profesorado toma en relación con aquello que espera que suceda, lo haya programado con anterioridad o lo decida sobre la marcha (p. 262).

Considera, asimismo, Pagés (2002), que tanto el currículo, como los futuros profesores, se ubican en dos ámbitos: el mundo de la reflexión y el análisis teórico, propio de la universidad, y el mundo de la práctica y de la acción (la escuela) y de ello se infiere que, efectivamente, la interrelación entre ambos mundos es fundamental para formar a un profesorado de nuevo cuño: un profesorado que aprende a partir de lo que ocurre en las aulas, de la experiencia. No obstante, resaltan Hernández y Pagés (2016) que:

La enseñanza y el aprendizaje de contenidos sociales e históricos deben considerar no solo la aplicación de actividades sino el contexto social, los intereses de la institución y de los padres de familia, así como las características del grupo donde trabajan. Para ello es importante que las finalidades se construyan y se definan en función de las necesidades y de los intereses de la comunidad donde está integrada la escuela preescolar (p.121).

La Didáctica de las Ciencias Sociales, es un campo cuyas investigaciones pueden considerarse recientes, como aprecia Prats (2002) cuando apunta la necesidad de construir un campo específico para ello, al encontrarse, a principios del siglo XXI, en una fase muy embrionaria, y puede añadirse que más carencias existen cuando se trata de la Educación Infantil (Miralles, Molina y Ortuño, 2011), si bien es cierto que el interés por la investigación, sobre Didáctica de las Ciencias Sociales, está encontrando un mayor impulso y se ha incrementado en los últimos años. Así se pone de manifiesto en el trabajo de Gómez-Carrasco, López-Facal, y Rodríguez-Medina (2019), cuando analizan la evolución de los artículos publicados en 34 revistas y concluyen que la producción científica se ha multiplicado por cinco entre 2007 y 2017. Por lo tanto, se puede argumentar que, actualmente, existe una fundamentación teórica que también debe incluirse en los estudios universitarios y, por ello, la asignatura en la que se centra este trabajo, está orientada a preparar al futuro profesorado y, como señalan Pastor, Alonso, Luna, Jiménez-Ridruejo, Rodríguez y Santisteban (2015), a conducir sus prácticas bajo el razonamiento de abordar la enseñanza de los saberes sociales, de modo que se consigan aprendizajes útiles y significativos para el alumnado. Por esa misma razón, es necesario aplicar distintas metodologías, por descubrimiento, activas, e interdisciplinares, que hagan posible la interactuación de todos estos conocimientos y también de manera experiencial, basados en la implicación del niño al que deben producir bienestar. Es decir, que toda estrategia de enseñanza/aprendizaje debe contar con metodologías que contengan una gran carga afectiva en una doble dirección: en primer lugar, entre el educador y el educando y, en segundo lugar, entre el educador y la materia que vaya a desvelar al niño (Pastor, 2004).

Cabe destacar que así se ha procurado al programar la asignatura y al intentar que las actividades prácticas, elaboradas por su alumnado, tanto en el aula, como en un trabajo de curso, respondan a esa diversidad metodológica que puede exigir cada caso particular y también a las 
características de edad, condiciones específicas, etc. que se presume en los niños y niñas de 3 a 5 años a las que se dirigen estas actuaciones.

\section{OBJETIVOS}

El principal objetivo es destacar la importancia del conocimiento del entorno en Educación Infantil y verificar que las Ciencias Sociales y su Didáctica proporcionan al alumnado las competencias apropiadas para ello, de cara a su futuro profesional.

Con ese interés se plantean los objetivos específicos siguientes:

Oe1. Analizar los aspectos básicos que marca el currículo del segundo ciclo de esta etapa de Educación Infantil.

Oe2. Exponer el ámbito de actuación de las Ciencias Sociales y su Didáctica.

Oe3. Poner de relieve tipos de actividades, propias de las Ciencias Sociales, que favorecen el conocimiento del entorno para el alumnado de Educación Infantil.

\section{MÉTODOS Y PROCEDIMIENTOS}

Las actividades realizadas, por el alumnado de dos grupos de tercer curso del Grado en Educación Infantil, constituyen la parte práctica del llamado trabajo final global de la asignatura Ciencias Sociales y su Didáctica en Educación Infantil, cuyo cuerpo conceptual pretende construir la base para la posterior aplicación de los conocimientos adquiridos, puesto que hoy sabemos que, sin renunciar a andar juntos un camino que contribuya a construir conocimiento y un aprendizaje asentado en el mapa cognitivo del alumnado, que cursa estudios universitarios, los docentes tenemos que procurarles herramientas para crear experiencias educativas que propicien el desarrollo de habilidades que, en Educación Infantil, hagan posible a los niños y niñas conocer, interpretar y participar de forma autónoma y responsable el entorno social y natural que les rodea.

Con ese interés se propone el trabajo final de la asignatura, que se desarrolla según el cronograma que presenta la Figura 3.

FIGURA 3. Procedimiento del trabajo

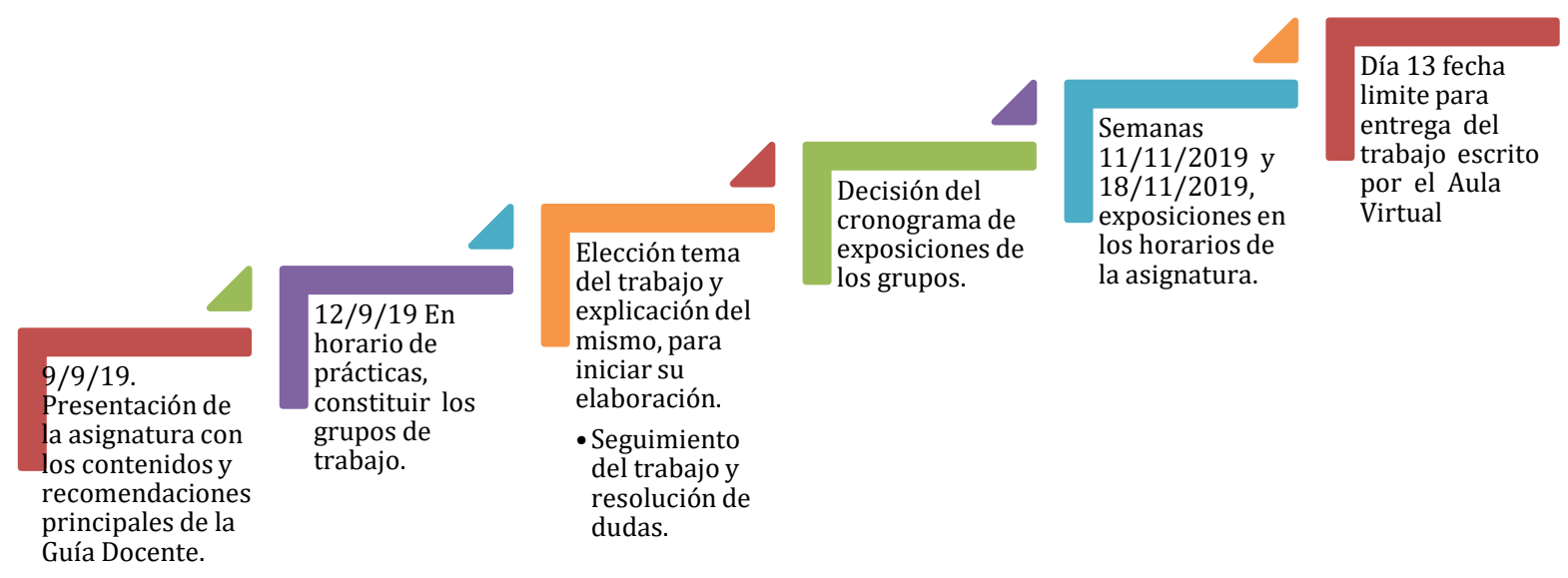

Fuente: Elaboración propia 
a) El agrupamiento

El número de participantes alcanza 102 y pertenecen a los Grupos 1 y 2 del tercer curso del Grado en Educación Infantil. En ambos grupos, se realizaron conjuntos, principalmente de cuatro personas, y han seguido las pautas indicadas en la tarea correspondiente.

Cabe advertir que la agrupación se realiza de forma voluntaria, pero procurando que nadie quede descolgado. En definitiva, se trata de aplicar un método de aprendizaje colaborativo en el que cada individuo toma un papel y ciertas responsabilidades dentro de su pequeño grupo. Cada miembro del equipo asume el compromiso sobre una parte del trabajo (pueden hacer una actividad cada uno), pero también es responsable del resultado del equipo completo, de ahí que se incluya una evaluación del grupo y una autoevaluación de cada miembro del grupo. Este planteamiento obedece a que, en un momento en el que la inclusión se ha convertido en un requisito imprescindible, en cualquier etapa educativa, el aprendizaje colaborativo es considerado, según (Vázquez, Méndez y Mendoza, 2017),

Como una construcción conjunta de significados y una relación e interacción basadas en el diálogo, la reflexión, el consenso, la participación, la comunicación y la responsabilidad compartida, cuya finalidad es crear espacios relacionales o de convivencia interpersonal que constituyan una comunidad educativa particular (p. 175).

b) Elección del tema central del trabajo

Una vez efectuada la organización del alumnado, con sus correspondientes grupos, se lleva a cabo la elección de la temática central del trabajo, teniendo en cuenta que debe versar sobre aspectos clave para el Área de Conocimiento el Entorno de Educación Infantil con intensificación en su Bloque 3. Cultura y vida en sociedad (Figura 4).

FIGURA 4. El Bloque 3 y sus objetivos.

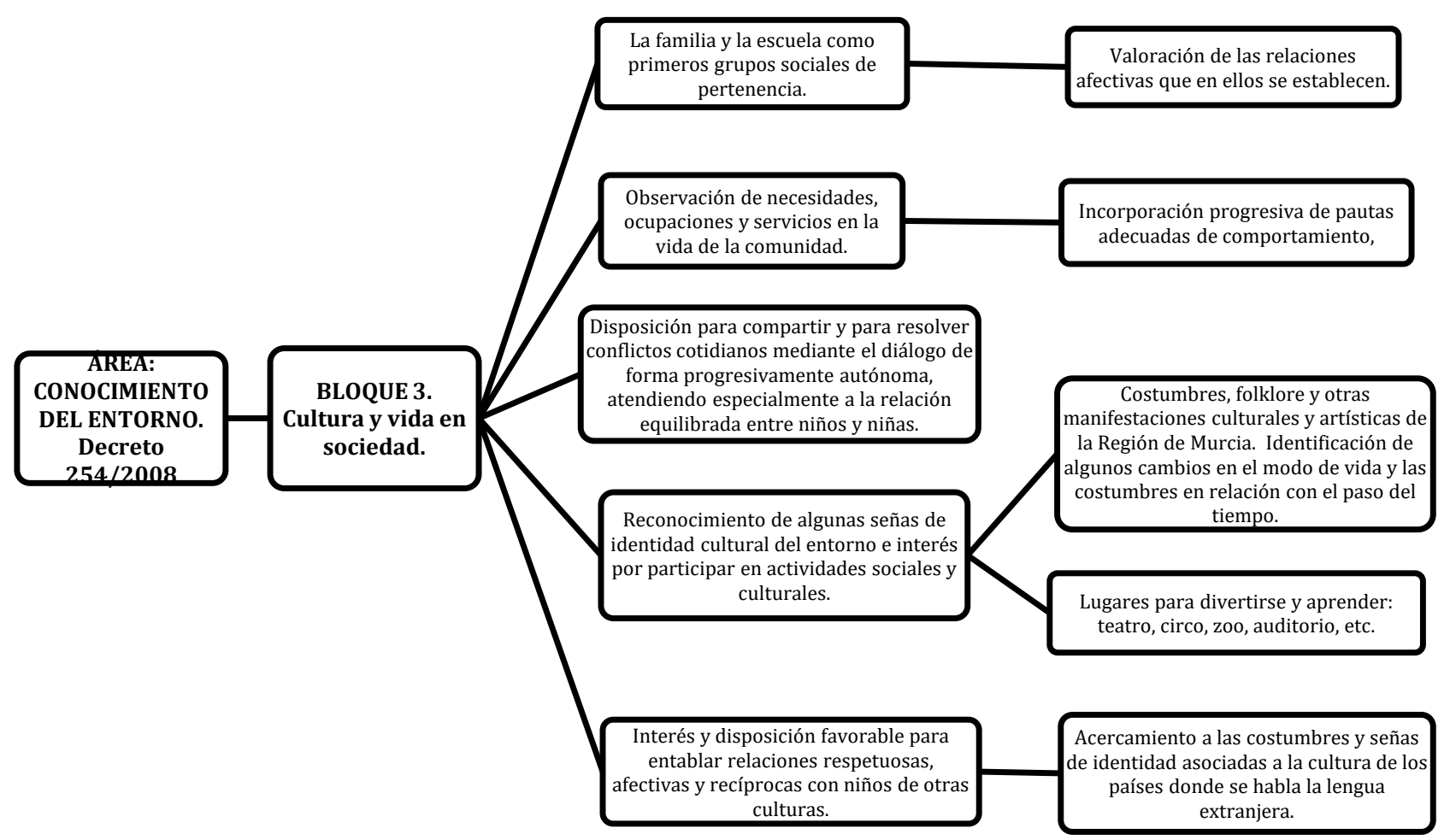

Fuente: Elaboración propia 
Esta elección obedece al interés por introducir actividades que favorezcan la resolución de problemas en los ámbitos cotidianos del alumnado de 3 a 5 años, como son la familia, la escuela y el entorno social y, cabe añadir que, considerando que el agrupamiento recomendado era de cuatro estudiantes, se establece también el número de cuatro actividades en las que se incluyan con claridad los objetivos, la metodología aplicada, la programación, los recursos para su puesta en práctica, los criterios de evaluación,...

c) Directrices sobre la estructura del trabajo.

Se acompañan en la propia Guía Docente de la asignatura y en la tarea creada en el Aula Virtual para este trabajo. Aquí se sintetizan en una tabla, que muestra los epígrafes obligatorios que debe incluir el documento final.

TABLA 1. Directrices para el trabajo de la asignatura

\begin{tabular}{ll}
\hline Estructura & - Portada (se recomienda la utilizada para los TFG de la Facultad de Educación), \\
& - Índice paginado, \\
& - Resumen y palabras clave, abstract y keywords \\
& - Introducción/justificación, \\
& - Marco teórico en el que aparezca un mínimo de cinco referencias/citas de bibliografía \\
& relevante para la temática tratada \\
& - Desarrollo del trabajo, con las cuatro actividades. \\
& - Conclusiones y autoevaluación. \\
\hline Formato & - Referencias. \\
\hline Extensión & La fuente de la letra podrá ser Times New Roman o Arial, tamaño 12 e interlineado 1,5. \\
\hline Referencias & La extensión máxima será de 20 páginas, pudiendo incluir, además, los oportunos Anexos. \\
& El trabajo deberá contar, asimismo, con las referencias necesarias en el cuerpo del trabajo, \\
& incluyendo tablas y figuras, así como las referencias finales de cuanto se haya utilizado como \\
& fuente. Todo ello siguiendo la normativa APA 6a edición. \\
\hline Entrega & Se entregará en Word y en Pdf por el Aula Virtual, en la fecha prevista a tal fin, facilitada en el \\
& cronograma de la asignatura. \\
\hline Evaluación & Mediante rúbrica \\
\hline
\end{tabular}

Fuente: Elaboración propia

Como se observa, la evaluación de la memoria escrita, se realiza mediante rúbrica y los criterios de calidad que se valoran se muestran, de forma sintetizada, en la Tabla 2.

TABLA 2. Criterios de calidad para valorar la información del trabajo escrito

1. Presentación del trabajo y redacción académica de los contenidos

2. Dominio de la materia.

3. Aplicación de búsqueda en bases de datos.

4. Capacidad de análisis y síntesis.

5. Saber relacionar los contenidos teóricos y prácticos en el trabajo.

Por lo que respecta a las actividades, incluidas en los trabajos, el formato de las mismas debía cumplir, al menos, la estructura que aparece en la Tabla 3.

TABLA 3. Apartados básicos de las actividades

- Título.

- Descripción.

- Edad a la que va dirigida.

- Objetivos.

- Metodología.

- Programación.

- Recursos.

- Competencias.

- Evaluación. 
Cabe señalar que el alumnado hacía una simulación del tipo de centro, de si existía algún caso de atención a la diversidad y a otras cuestiones de posibles particularidades. Generalmente, al tratarse de una asignatura de tercer curso, utilizaban para este criterio la experiencia adquirida en las prácticas ya realizadas en los centros escolares.

Por otro lado, en el diseño de las actividades se dio libertad, siempre cumpliendo los apartados básicos exigidos, y ello ha repercutido en presentaciones con diversidad de formatos que incluían tablas o epígrafes de texto más desarrollado.

d) Elección del método o métodos para conseguir los objetivos de las actividades.

Precisamente por tratarse de un trabajo universitario, e ignorar la forma en la que podrá utilizarse con niños y niñas de los centros escolares, se indicaron varias opciones metodológicas, siempre que el objeto principal de análisis fuese la problemática observada en la escuela, la familia y/o el entorno social, aspectos estos que se tratan en los contenidos de la asignatura.

Entre los métodos que podían aplicarse se encuentran el aprendizaje por descubrimiento y construcción (Bruner, 1973), que apuesta por un aprendizaje que trasciende la mera enseñanza a base de información, algo en lo que coinciden los ya citados Miralles y Rivero (2012), y busca aprender a aprender y a resolver problemas.

Bruner (1973) también muestra su atención por el aprendizaje basado en proyectos (ABP), en el que, según Restrepo (2005), el papel del docente es el de orientar y, a partir de exponer problemas o situaciones problemáticas, realizar sugerencias sobre fuentes de información y colaborar con las necesidades del aprendiz. Bajo estas premisas, las experiencias, efectuadas en las aulas, suelen resultar satisfactorias siempre que el método siga unas reglas básicas en la secuencia didáctica, desde la pregunta inicial a resolver, a las decisiones, la reflexión y la revisión crítica de lo realizado. En este sentido, Restrepo (2005), estima apropiado "que los problemas simulados que se utilizan para promover el aprendizaje deben ser progresivamente abiertos, no estructurados o brunerianos, para que el estudiante agudice su habilidad de búsqueda” (p.11). Garrigós y Valero (2012), por su parte, consideran que, con la aplicación de métodos de aprendizaje de este tipo, mejora sustancialmente la motivación del alumnado y hacen que se esfuerce más.

No parecía oportuno excluir, de las posibilidades de elección, el aprendizaje significativo de Ausubel (1983) quien plantea lo siguiente:

El aprendizaje del alumnado depende de la estructura cognitiva previa que se relaciona con la nueva información, debe entenderse por "estructura cognitiva", al conjunto de conceptos, ideas que un individuo posee en un determinado campo del conocimiento, así como su organización. (p. 1).

Ausubel, Novak y Hanesian (1976), ya habían considerado que la esencia del proceso del aprendizaje significativo reside relacionar las ideas de manera sustancial y no al pie de la letra, de forma que el alumno pueda establecer una vinculación con algún aspecto esencial de su estructura de conocimientos, como puede ser una imagen, un símbolo, un contexto, ...

Como muchas teorías, el aprendizaje significativo ha encontrado detractores y defensores, pero pasados los años sigue estando vigente porque, como dice Moreira (2017), tiene por objeto "la adquisición de nuevos conocimientos con significado, comprensión, criticidad y posibilidades de usar esos conocimientos en explicaciones, argumentaciones y solución de situaciones-problema, e incluso nuevas situaciones” (p. 2). Precisamente por ello, es apropiado aplicar la filosofía del aprendizaje significativo en un contexto que, con frecuencia, busca más entrenar para responder correctamente test, que lo indicado verdaderamente en esta teoría (Moreira, 2017).

e) Seguimiento del proceso.

Como la asignatura se desarrolla en el primer cuatrimestre, la puesta en práctica de los resultados del trabajo será posible en las prácticas escolares posteriores, pero puede considerarse un 
paso preparatorio para esa función docente en prácticas y así se ha transmitido ya en casos concretos por parte del alumnado. No obstante, como también apunta López (2014), somos conscientes de la dificultad que encuentran, en muchas ocasiones, los futuros docentes para aplicar métodos innovadores en las aulas y más todavía cuando se trata de las de Educación Primaria.

En el caso concreto analizado, el horario semanal de la asignatura, diferenciando con claridad las horas de teoría y de prácticas, en las que se dividía el gran grupo, ha hecho posible que el alumnado haya ido planteando cualquier duda en la propia aula y esto ha facilitado una progresión significativa para el desarrollo de las cuestiones de formato, utilización de las referencias según normas APA, o cualquier otra dificultad. Los grupos han estado trabajando de forma colaborativa y las sesiones se desarrollaron con muy buena sintonía entre todos los participantes.

e) Exposición pública de los resultados del trabajo.

Los resultados se exponen ante todo el grupo, habiendo establecido para ello, como se indica en el correspondiente cronograma, las últimas semanas de clase, anteriores al periodo de exámenes. En concreto, se realizaron las semanas del 11 y 18 de noviembre de 2019, quedando la semana del 25 en adelante reservada para posibles incidencias.

La organización de las exposiciones se efectuó consensuando fechas y distribución horaria. El tiempo para la presentación se estipuló en 20 minutos y todo discurrió de manera satisfactoria e incluso distendida, en no pocas ocasiones, puesto que algunas actividades, sobre todo las basadas en juegos, se llevaban a efecto con la participación de otros compañeros y compañeras de clase. Sin duda, además de un aprendizaje muy valioso, de cara a su futuro profesional, el alumnado pudo comprobar con esta experiencia las múltiples posibilidades que la Didáctica de las Ciencias Sociales proporciona para etapa de Educación Infantil.

f) Evaluación y Criterios de calidad.

Entre los principios, que guían la labor docente en Educación Infantil, se encuentran los aprendizajes significativos y esto mismo puede lograrse para el alumnado de Grado si se consigue establecer múltiples relaciones entre los conceptos aprendidos para que, de manera activa, cada estudiante construya y amplíe sus conocimientos, creando conexiones entre lo que ya sabe y lo nuevo que ha aprendido y dando significado a dichas relaciones. Asimismo, se evalúa el cumplimiento del compromiso asumido con el grupo y, también, con toda la clase, puesto que compartir sus hallazgos, su creatividad, representa un beneficio común. Por esa misma razón, se aplica, de igual modo, el formato de la autoevaluación, junto a los criterios previamente definidos en la rúbrica del trabajo.

La valoración del conjunto se realiza desde dos perspectivas, la que resulta del trabajo escrito y la de la exposición ante toda la clase, puesto que es también, en dicha presentación, cuando se muestran materiales elaborados por el alumnado y se practican algunas de las actividades.

\section{RESULTADOS}

En relación a lo esperado, para el Área de Conocimiento del entorno, se considera que la intervención educativa tendrá como objetivo el desarrollo de capacidades a partir de la observación de forma activa del entorno del alumnado; la relación con los demás; la interrelación con grupos sociales diversos; el acercamiento a los componentes básicos del medio natural, animal y vegetal, con la finalidad de desarrollar actitudes de respeto y valoración y un acercamiento a las manifestaciones culturales que les son más próximas y constituyen el patrimonio identitario del alumnado de esta etapa.

De ello se desprende que las actividades prácticas deben centrarse en conseguir tales propósitos y así ha sido en el caso que aquí se expone. Como puede observarse en la gráfica (Figura 5) 
FIGURA 5. Distribución porcentual de los temas trabajados en las actividades.

PRINCIPALES TEMAS DE LOS TRABAJOS

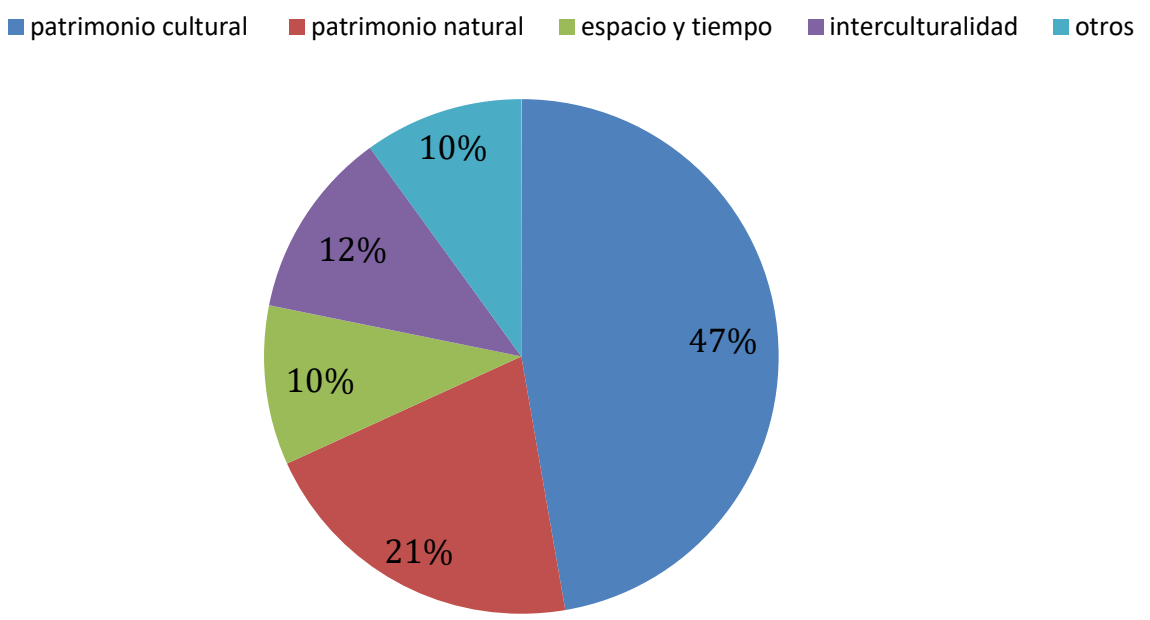

Fuente: Elaboración propia

Un mayor desglose permite destacar que, dentro de los trabajos, que se han interesado por el patrimonio cultural de la Región de Murcia, lo han hecho desde diversas perspectivas, puesto que han realizado actividades de acercamiento al Arte Rupestre, presente en diferentes municipios y, como en otras Comunidades Autónomas del levante español, goza de la Declaración de Patrimonio de la Humanidad por la Unesco. También han utilizado aspectos identitarios relacionados con la Huerta de Murcia, sus características de ritos y costumbres, los bailes, su vestimenta, o la gastronomía típica de este enclave de la Región de Murcia. Las visitas a museos, de diversas características, tipos de fondos y exposiciones, igualmente han resultado de interés a la hora de diseñar actividades y otro tanto ha ocurrido con la identificación de los oficios y su contribución a la sociedad, cuya representación tan heterogénea va conociendo el alumnado en su experiencia vital cotidiana de forma paulatina.

Además, se han realizado actividades centradas en las fiestas y otras manifestaciones culturales, puesto que enlazan con lo mostrado en el currículo de esta etapa, por presentar como uno de sus objetivos el reconocimiento de algunas señas de identidad cultural del entorno y el interés por participar en actividades sociales y culturales.

Sin duda, en cada pueblo es fácil encontrar un momento para confraternizar en este tipo de eventos, incluso desde el nacimiento, pero la Región de Murcia vive particularmente acontecimientos muy relevantes en la primavera y se produce un encuentro de numerosos habitantes de la Comunidad Autónoma, tanto en el llamado Bando de la Huerta, como en el Entierro de la Sardina. Por ello, en la etapa de Educación Infantil es muy frecuente que el alumnado haya experimentado esa vivencia en la capital regional, lo mismo que en otros municipios como Cartagena, Lorca o Caravaca, lo hacen con sus eventos festivos más significativos y así hasta los 45 que componen la Comunidad Autónoma, que cuentan con diferentes celebraciones cuyos desfiles, recreaciones históricas y otros tipos de acontecimientos, poseen un arraigo popular e identitario muy notable. En este sentido, es preciso advertir que el tema: "Manifestaciones culturales a lo largo del tiempo en la Región de Murcia”, forma parte de los contenidos de la asignatura a la que nos referimos aquí y ello permite realizar un recorrido sobre los principales recursos culturales de esta Comunidad Autónoma y descubrir los pueblos y ciudades que destacan en la misma por su legado patrimonial, tanto material como inmaterial. Y, algo que es preciso indicar es que, efectivamente, 
para nuestros estudiantes ha sido, en muchos casos, un auténtico descubrimiento comprobar la abundancia y calidad de dicho patrimonio, pero también es importante señalar que ha sido un acicate para llevar a cabo una reflexión sobre la importancia de conocer de dónde venimos, cuáles son las señas de identidad que caracterizan a la Región de Murcia, cómo debemos aprender a valorarlo y conservarlo para generaciones futuras y, de igual modo, qué compartimos con los que han llegado de otros territorios, porque abordar los posibles problemas que pueden suscitarse entre diferentes culturas, resulta mucho más sencillo y efectivo identificando lo que nos une y fomentando una educación basada en la interculturalidad.

Respecto al patrimonio natural, han sido mencionados distintos tipos de fauna, de arbolado y flora en general, así como trabajar las estaciones del año, los ríos, el mar, etc. Pueden incluirse aquí las actividades que han realizado diseños a favor de la concienciación con el desarrollo sostenible, la situación del Mar Menor, el reciclado de distinos elementos cotidianos, el uso racional del agua, evitar el despilfarro de recursos en general y el consumo responsable de los mismos, han constituido algunas de las aportaciones relacionadas con la sensibilidad medioambiental que debe construirse desde esta etapa educativa, no solo por parte de escuela, sino de la familia y todo el grupo social de pertenencia.

Las cuestiones espacio temporales, a partir de juegos relacionados con las rutinas diarias, los árboles genealógicos, las historias personales y familiares; la noción de sucesión en el ámbito familiar, los días de la semana, los itinerarios, juegos de localización, etc., han sido otras aportaciones que se han alternado con actividades que contribuyen a mejorar la antes mencionada interculturalidad, por ejemplo se han confeccionado materiales con las vestimentas propias de distintos países, banderas, platos típicos, en los que aparecen ingredientes comunes a buena parte gastronomías populares, palabras de diferentes idiomas, etc. En definitiva, todo un abanico de actividades y de recursos didácticos, elaborados por los estudiantes, con el objetivo de implementarse en las aulas de Educación Infantil.

\section{CONCLUSIONES}

Considerando el principal objetivo de este trabajo, de lo expuesto en el mismo se infiere que la asignatura Ciencias Sociales y su Didáctica, del Grado de Educación Infantil, contribuye a que los futuros docentes adquieran las capacidades apropiadas para desarrollar, de forma satisfactoria, el proceso de enseñanza-aprendizaje para el alumnado de dicha etapa educativa. Esta aseveración se fundamenta en los resultados observados, tanto en las memorias de los trabajos finales, que incluyen una justificación teórica, de las decisiones estratégicas aplicadas en las actividades incluidas en los mismos, como en la prueba de conocimientos adquiridos de la asignatura, que se concreta en un examen final, con gran peso en la parte teórica, cuya valoración representa un $60 \%$ de la nota total.

Respecto a los objetivos específicos, el Oe1 se ha considerado en el planteamiento de toda la asignatura, cuyas competencias se han indicado en la introducción/justificación, y así se ha puesto de relieve también a lo largo de este artículo, puesto que es la base en la que se sustenta buena parte de cuanto se ha justificado, algo que siempre se ha hecho procurando destacar aquellos aspectos más vinculados a las Ciencias Sociales, en consonancia con la intención del Oe2. Por último, el Oe3 ha ocupado buena parte de los argumentos y resultados obtenidos, por lo que puede estimarse totalmente conseguido al exponer, aunque de forma sintetizada, la abundancia de actividades distintas y la profusión de recursos didácticos incluidos en las mismas.

Es evidente que el producto final de esta experiencia no puede exhibirse aquí y que será el alumnado de la asignatura quien, en un futuro, comprobará si el esfuerzo realizado ha sido suficientemente enriquecedor y si ha constituido un aprendizaje significativo en vistas a sus próximas prácticas docentes. 
También hay que advertir que, para esta aportación, se ha evitado utilizar los materiales elaborados por los participantes en la asignatura, lo mismo que se ha preservado su identidad por razones obvias de protección de datos.

Desde el punto de vista de la discusión de los resultados, el punto de partida es reconocer que para los estudiantes, futuros docentes, no siempre resulta motivador el aprendizaje de una base conceptual y metodológica de cualquier disciplina, pero es necesario asumir que la formación en Didáctica de las Ciencias Sociales debe fundamentarse en los conocimientos de las ciencias que son sus referentes, puesto que, como apunta Pagés (1993), darán coherencia para construir modelos para el análisis y la intervención en la práctica educativa.

Sigue esta línea Santisteban (2008), cuando refiere que el profesorado debe formarse "poniendo el acento en los mecanismos o procesos de construcción científica de las ciencias sociales” (p. 82) y que la Didáctica de las Ciencias Sociales tiene que favorecer una reflexión sobre el papel que estas desempeñan en la formación de las personas para que adquieran una cultura democrática, a través de la enseñanza de la Historia, la Geografía u otras ciencias sociales.

También Santisteban (2008) considera que "la didáctica de las ciencias sociales debe construir el conocimiento describiendo e interpretando la relación entre la práctica y la teoría” (p. 82). Esto permite enlazar con lo expuesto por Miralles y Rivero (2012) cuando ven al docente como facilitador de los aprendizajes mediante diferentes estrategias y con Pagés (2002), que se refiere al mundo de la reflexión y el análisis teórico, propio de la universidad, y el mundo de la práctica y de la acción (la escuela).

Por todo ello es oportuno considerar que los resultados de esta aportación, reflejan que, con el desarrollo de la asignatura, se ha experimentado un cambio sustancial, sobre la misma y sus contenidos, en la percepción del alumnado, puesto que ha podido comprobar cómo los discursos y enseñanzas propios de las Ciencias Sociales son tan fácilmente reconocibles en el entorno habitual, en este caso la Región de Murcia, y permiten explicar las relaciones con el espacio y el tiempo que conforman la vida en sociedad, su cultura, los usos y costumbres del entorno del alumnado.

Por su parte, el trabajo de curso representa la búsqueda de esa interrelación, anotada por Pagés (2002), entre la reflexión y el análisis teórico, cuando el alumnado lo plasma en la introducción/justificación y el marco teórico y la práctica que debe llevar a la escuela mediante actividades y recursos didácticos apropiados.

En las Tablas 1 y 2, se han mostrado, por un lado, los requisitos que deben cumplirse para tratar didácticamente los contenidos de la asignatura y aplicarlos a estos trabajos académicos; por otro lado, los criterios de evaluación. Así, a modo de ejemplo, se cita una actividad que forma parte del proyecto más amplio respecto a los acontecimientos representados en las Fiestas de Primavera de la ciudad de Murcia. Dicha actividad introduce a los niños y niñas en un evento, conocido como Batalla de las Flores, que se contextualiza en el tiempo y en un espacio que resulta familiar para el alumnado de Educación Infantil y se desarrolla mediante la siguiente estructura: 1. Objetivos, 2. Descripción, 3. Temporalización, 4. Metodología, 5. Recursos didácticos, 6. Competencias, 7. Evaluación, 8. Reflexión sobre la actividad.

Es decir, no se trata meramente de hacer actividades, sino que estas deben realizarse en consonancia con lo que se pretende enseñar, con relación al conocimiento del entorno y con los objetivos del currículo de la etapa de Educación Infantil, porque todo ello se encuentra en la esencia del descubrimiento paulatino que van a experimentar los niños y niñas $\mathrm{y}$, por lo tanto, es necesario que los docentes les acompañen y guíen en ese tránsito.

Cabe recordar que los objetivos de la etapa persiguen conseguir, entre otras cuestiones, aprender a mirar y leer el espacio, su evolución, saber cuántos y quiénes son sus habitantes, a qué se dedican y a qué obedece la existencia de una profesión determinada, su importancia para la sociedad, etc.

De lo que no hay duda es de que, tanto la metodología colaborativa, con la que se han llevado a cabo los trabajos de curso, como las actividades incluidas en ellos, han sido valorados de forma 
muy satisfactoria por la práctica totalidad de los 102 participantes. En este sentido, solamente basta citar un ejemplo de las frases más comunes que se encuentran en las autoevaluaciones de los trabajos: "hemos relacionado los contenidos teóricos y prácticos vistos en la asignatura, de manera que nos hace comprender de forma más fácil y motivadora al realizar el trabajo de curso de manera grupal”.

En todos los casos se valoró la dinámica conseguida en el aula y se reconoció que se habían logrado actitudes fundamentales de respeto a la opinión del otro y a la búsqueda del consenso para mejorar el resultado final. Sin renunciar a la confidencialidad y omitiendo los nombres, se acompaña un ejemplo literal de las autoevaluaciones y una tabla de valoración de grupo como anexos. De ello se desprende que la valoración propia ha sido alta de forma bastante generalizada, pero quien ha tenido alguna dificultad para llevar el ritmo del grupo también lo ha indicado y, con relación al grupo, resulta relevante la homogeneidad en considerar que todos sus integrantes han colaborado por igual. Así se desprendía también de las intervenciones realizadas al exponer los trabajos, aquí la sintonía y el apoyo entre los participantes, tanto los que realizaban la presentación, como el resto de la asamblea que recibía con interés y disfrutaba de las actividades de los demás, ha resultado la tónica más común. Como dicen Gómez-Ruiz y Quesada (2017) este formato de evaluación se negocia entre docentes y estudiantes, la responsabilidad es compartida y el diálogo es imprescindible. Además, favorece el aprendizaje autónomo y la autorregulación.

Todo esto no tendría tanta importancia, desde el punto de vista académico, si no estuviese acompañado por la superación, en un porcentaje significativo de estudiantes, del examen final de la asignatura que, fundamentalmente, aborda cuestiones teóricas, como ya se ha dicho. De ahí que la conclusión general, de su desarrollo, sea satisfactoria.

\section{Referencias}

Alonso, A. (2007). Hacia una nueva concepción de la Educación Infantil: jalones históricos y antecedentes para la puesta en marcha de la Reforma. Anuario de Pedagogía, (9) 11-46.

Aranda, A. Ma . (2003). Didáctica del Conocimiento del medio social y cultural en Educación Infantil. Madrid: Síntesis.

Ausubel, D., Novak, J. D., y Hanesian, H. (1976). Significado y aprendizaje significativo. Psicología educativa: un punto de vista cognoscitivo, 53-106. Recuperado de: https://cmapspublic2.ihmc.us/rid=1J3D72LMF-1TF42P4-

PWD/aprendizaje\%20significativo.pdf

Ausubel, D. (1983). Teoría del aprendizaje significativo. Fascículos de CEIF, 1, 1-10.

Blanco, P., De la Calle, M., Fernández, R. (Et al). (2008). Una propuesta adaptada al espacio europeo de educación superior. En: R. Ávila, A. Cruz y M. Díez (eds.), Didáctica de las Ciencias Sociales, Currículo Escolar y Formación del Profesorado. La Didáctica de las Ciencias Sociales en los nuevos planes de estudio (pp. 113-128). AUPDCS.

Bruner, J. S. (1973). Beyond the information given. Nueva York: Norton

Bruner, J. (1997). La educación, puerta de la cultura. Madrid: Aprendizaje-Visor

Egan, K. (1991). La comprensión de la realidad en la educación infantil y primaria (Vol. 19). Madrid: Ediciones Morata.

García, M., Villegas, M. y González, F. (2015). La noción del espacio en la primera infancia: un análisis desde los dibujos infantiles. Revista Paradigma, 36(2), 225-245.

García-Ruiz, R. (2013). Enseñar y aprender en Educación Infantil a través de proyectos (Vol. 17). Ed. Universidad de Cantabria.

Garrigós, J. y Valero, M. (2012). Hablando sobre Aprendizaje Basado en Proyectos con Julia. Revista de Docencia Universitaria. REDU, 10(3) 125-151.

Gervilla, Á. (2016). El currículo de educación infantil: aspectos básicos (Vol. 57). Madrid: Narcea Ediciones. 
Gomez-Carrasco, C. J., López-Facal, R. y Rodríguez-Medina, J. (2019). La investigación en Didáctica de las Ciencias Sociales en revistas españolas de Ciencias de la Educación. Un análisis bibliométrico (2007-2017). Didáctica de las Ciencias Experimentales y Sociales, 37, 67-88. DOI: 10.7203/dces.37.14440

Gómez-Ruiz, M.A. y Quesada, V. (2017). Coevaluación o Evaluación Compartida en el Contexto Universitario: La Percepción del Alumnado de Primer Curso. Revista Iberoamericana de Evaluación Educativa. 10(2), 9-30. DOI: 10.15366/riee2017.10.2.001

Hernández, L. y Pagés, J. (2016). ¿Cómo enseñar historia y ciencias sociales en la educación preescolar? Revista mexicana de investigación educativa, 21(68), 119-140.

López, E. (2014). Aprendizaje Basado en Proyectos para el desarrollo de las competencias profesionales del maestro: una propuesta de innovación docente desde la Didáctica de las Ciencias Sociales. Didáctica de las Ciencias Experimentales y Sociales, 29, 25-41. DOI: 10.7203/dces.29.3775

López, I. (2010). El juego en la educación infantil y primaria. Autodidacta, 1(3), 19-37.

López, R. (2003). La enseñanza de la historia, más allá del nacionalismo. En J.J. Carreras y C. Forcadell (Eds.), Usos públicos de la historia (pp. 223-256). Madrid: Marcial Pons-Prensas Universitarias de Zaragoza.

Mateos, J. (2001). Genealogía del código pedagógico del entorno. Aula, 13, 19-35.

Marín-Díaz, V. y Sánchez-Cuenca, C. (2015). Formación en valores y cuentos tradicionales en la etapa de educación infantil. Revista Latinoamericana de Ciencias Sociales, Niñez y Juventud $R L C S N J, 13(2), 1093-1106$.

Miralles, P. y Rivero, P. (2012). Propuestas de innovación para la enseñanza de la historia en Educación Infantil. Revista electrónica interuniversitaria de formación del profesorado, 15(1), 81-90.

Miralles, P., Molina, S. y Ortuño, J. (2011). La investigación en Didáctica de las Ciencias Sociales. Educatio siglo XXI, 29(1), 149-174.

Moreira, M. A. (2017). Aprendizaje significativo como un referente para la organización de la enseñanza. Archivos de Ciencias de la Educación, 11(12), e029. DOI: $10.24215 / 23468866 \mathrm{e} 029$

Pagès, J. (1993). Psicología y didáctica de las ciencias sociales. Infancia y aprendizaje, 16(62-63) 121-151.

Pagès, J. (2002). Aprender a enseñar historia y ciencias sociales: el currículo y la didáctica de las ciencias sociales. Pensamiento educativo, 30(1) 255-269.

Pastor, M. M. (2004). Estrategias y métodos didácticos para la enseñanza/aprendizaje de las Ciencias Sociales. En Ma C. Domínguez (Coord.), Didáctica de las Ciencias Sociales (pp. 147-203). Madrid: Pearson Prentice Hall.

Pastor, M. M., Alonso, J. I., Luna, G., Jiménez-Ridruejo, G., Rodríguez, T. M., y Santisteban, A. (2015). Propuestas didácticas de carácter interdisciplinar para la enseñanza/Aprendizaje del espacio y el tiempo en la educación infantil. Didácticas Específicas, 13, 87-104.

Prats, J. (2002). Hacia una definición de la investigación en didáctica de las ciencias sociales. Enseñanza de las Ciencias Sociales. Revista de investigación, 1, 81-89.

Pluckrose, H. (2002). Enseñanza y aprendizaje de la Historia. Colección Pedagogía. Educación Infantil y Primaria. (Cuarta Edición) Madrid: Ediciones Morata S. L.

Restrepo, B. (2005). Aprendizaje basado en problemas (ABP): una innovación didáctica para la enseñanza universitaria. Educación y educadores, 8, 9-20.

Rivero, P. y Pelegrín, J. (2019). ¿Qué Historia consideran relevante los futuros docentes de Educación Infantil? Cadernos de Pesquisa, 49(172), 96-119.

Romero, J. y Luis, A. (2008). El conocimiento sociogeográfico en la escuela: las tensiones inherentes a la transmisión institucionalizada de cultura y los dilemas de la educación para la 
democracia en este mundo globalizado. Revista Scripta Nova Revista Electrónica de Geografía y Ciencias Sociales, 12(270).

Santisteban, A. (2008). La formación inicial del profesorado de Educación Primaria para enseñar Ciencias Sociales: futuro presente. En R. M. Ávila, M. A. Cruz y M. C. Díez (eds.), Didáctica de las Ciencias Sociales, Currículo Escolar y Formación del Profesorado. La Didáctica de las Ciencias Sociales en los nuevos planes de estudio (pp. 79-99) Jaén: AUPDCS.

Schiller, P. y Rossano, J. (1993). 500 actividades para el currículo de educación infantil (Vol. 23). Madrid: Narcea Ediciones.

Souto, X. M. y García. D. (2016). La geografía escolar ante el espejo de su representación social. Didáctica Geográfica, 17, 177-201.

Vázquez, M. G., Méndez, J. M. y Mendoza, F. M. (2017). Educación inclusiva y aprendizaje colaborativo en el aula: un estudio de la práctica docente universitaria. Revista de Educación Inclusiva, 8(3), 171-187.

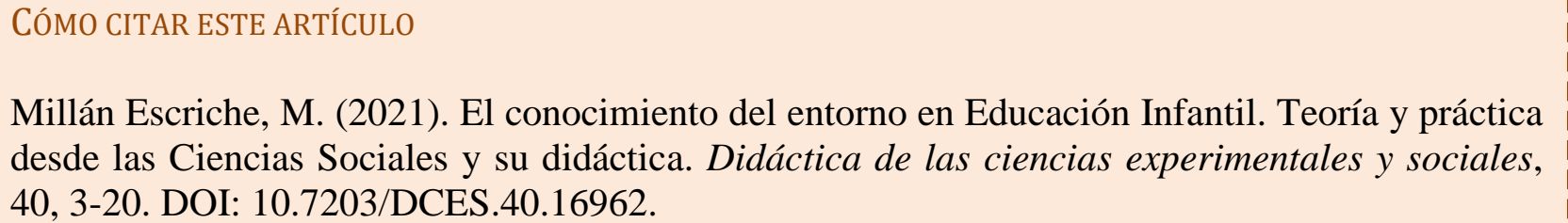




\section{ANEXOS}

\section{ANEXO 1. Ejemplo evaluación individual}

En este apartado cada alumna expondrá de forma más detallada su parte del trabajo realizado y cómo se ha sentido con el desarrollo del mismo y con sus compañeras.

- A.1: Considero que he trabajado como es debido, aportando ideas y cooperando con mis compañeras tanto en la elaboración del documento como en lo que a creación de material se refiere. Por último, cabe destacar que el clima de trabajo ha sido favorable y ha posibilitado que demos lo mejor de nosotras, complementándonos unas a otras y consiguiendo una creación basada en el consenso, la escucha activa y la ayuda mutua. Por mi parte, destaco mi tesón y constancia que, a mi modo de verlo, son ingredientes fundamentales para obtener buenos resultados.

- A.2: Durante la realización de este trabajo, he trabajado con constancia y he propuesto ideas al resto de mis compañeras. En general, hemos trabajado conjuntamente aportando ideas y mejorando ideas de otras. En mi caso, considero que todas hemos mantenido una actitud empática y solidaria entre nosotras, trabajando de manera igualitaria y lo más importante ayudándonos entre nosotras, siempre dentro de un clima sano y colaborativo.

- A.3: En este trabajo, aunque su mayor parte la hemos hecho de forma grupal, aportando ideas y redactando los contenidos en consenso, también hemos tenido parte individual, llevada a cabo desde casa. La elaboración no ha sido demasiado costosa, pues es un tema que nos interesaba mucho y que está presente en nuestro día a día. Creo que mi trabajo ha sido el adecuado, mostrando una actitud siempre positiva y comprometida. Al igual que el de mis compañeras. En conjunto formamos un grupo bastante productivo.

- A.4. Tras todo el tiempo y dedicación puesto en este trabajo, puedo llegar a decir que me ha dejado un buen sabor de boca. Pues desde un principio las expectativas que tenía eran mucho más bajas, ya que la elección de las actividades nos ha supuesto un campo muy amplio y a la vez muy reducido. Se debe a que la metodología que hemos pretendido llevar es doble, de aprendizaje por descubrimiento y significativo. Además, la búsqueda de información en más de una fuente, en ocasiones ha hecho que la extracción de información haya sido más compleja, pero sin duda mucho más rica. Por otra parte, he tenido muy buena relación con mis compañeras, lo cual ha hecho que me sienta cómoda y segura a la hora de exponer mis ideas. Desde mi punto de vista, aunque todas coincidimos, hemos colaborado y trabajado de manera equitativa, apoyándonos las unas a las otras y por supuesto escuchándonos. En resumen, nos hemos esforzado y creo que hemos hecho un gran trabajo del cual hemos aprendido bastante, sobre todo de cara a nuestro futuro.

\section{ANEXO 2. Ejemplo de tabla de coevaluación}

Coevaluación de las integrantes del grupo

\begin{tabular}{cccccc}
\hline Evaluadores & Criterio 1 & Criterio 2 & Criterio 3 & Criterio 4 & Criterio 5 \\
\hline 1. & 1.7 & 2 & 2 & 1.7 & 2 \\
2. & 2 & 1.7 & 1.7 & 2 & 2 \\
3. & 2 & 2 & 2 & 1.7 & 1.7 \\
4. & 1.7 & 2 & 1.7 & 2 & 2
\end{tabular}

Valoración de las componentes del grupo siguiendo los criterios expuestos anteriormente (elaboración propia, 2019).

Criterios están evaluados del 0 (siendo este, la nota más baja) hasta un 2 (siendo la más alta).

La explicación sobre el formato de coevaluación entre las alumnas del grupo sigue los siguientes criterios de cooperación:

1. Que se haya cooperado tanto de forma grupal como individual en la realización del documento.

2. Ningún miembro del grupo se haya opuesto a quedar en horarios extraoficiales para realizar el documento.

3. El trabajo realizado ha sido continuo, dinámico y sistemático.

4. Muestra de interés por la asignatura y el trabajo en grupo.

5. Los problemas se han resuelto de forma pacífica, explicando al grupo las causas de estos. 